\title{
Implementation of INHERET, an Online Family History and Cancer Risk Interpretation Program for Primary Care and Specialty Clinics
}

Lynn A. McCain, MHSA ${ }^{1,2}$; Kara J. Milliron, MS, CGC ${ }^{2,3}$; Amanda M. Cook, BA²; Robert Paquette, BS²; Jasmine B. Parvaz, MD, PhD ${ }^{4}$; Susan D. Ernst, MD ${ }^{5}$; Anne L. Kittendorf, MD ${ }^{6}$; Diane M. Harper, MD ${ }^{6}$; Philip Zazove, MD ${ }^{6}$; Jim Arthurs, BSEE ${ }^{2,7}$; Jerry A. Tippie, BA ${ }^{2}$; Bailey Hulswit, MS, CGC ${ }^{3}$; Lee F. Schroeder, MD, PhD ${ }^{1,2}$; David F. Keren, MD ${ }^{1,2}$; and Sofia D. Merajver, MD, PhD $2,3,8$

\begin{abstract}
Background: Individuals at increased risk for cancer are ascertained at low rates of $1 \%$ to $12 \%$ in primary care (PC). Underserved populations experience disparities of ascertainment, but data are lacking. INHERET is an online personal and family history tool to facilitate the identification of individuals who are eligible, according to guidelines, to be counseled on germline genetic testing and risk management. Patients and Methods: INHERET data entry uses cancer genetics clinic questionnaires and algorithms that process patient data through NCCN Clinical Practice Guidelines in Oncology and best practice guidelines. The tool was tested in silico on simulated and retrospective patients and prospectively in a pilot implementation trial. Patients in cancer genetics and in PC clinics were invited to participate via email or a card. Informed consent was completed online. Results: INHERET aimed to integrate patient data by algorithms based on professional and best practice guidelines to elicit succinct, actionable recommendations that providers can use without affecting clinic workflow or encounter length. INHERET requires a 4th-grade reading level, has simple navigation, and produces data lists and pedigree graphs. Prospective implementation testing revealed understandability of $90 \%$ to $100 \%$, ease of use of $85 \%$, and completion rates of $85 \%$ to $100 \%$. Physicians using INHERET reported no added time to their encounters when patients were identified for counseling. In a specialty genetics clinic, INHERET's data were input, on average, within 72 hours compared with 4 to 6 weeks through standard care, and the queue for scheduling patients decreased from 400 to fewer than 15 in $<6$ months. Conclusions: INHERET was found to be accessible for all education and age levels, except patients aged $>70$ years, who encountered more technical difficulties. INHERET aided providers in conveying high-risk status to patients and eliciting appropriate referrals, and, in a specialty clinic, it produced improved workflows and shortened queues.
\end{abstract}

J Natl Compr Canc Netw 2022;20(1):63-70 doi: $10.6004 /$ jnccn.2021.7072

\footnotetext{
${ }^{1}$ Department of Pathology, University of Michigan Medical School; ${ }^{2}$ INHERET, Inc.; ${ }^{3}$ Department of Internal Medicine, University of Michigan Medical School; ${ }^{4}$ University Health Services, University of Michigan; ${ }^{5}$ Department of Obstetrics and Gynecology, ${ }^{6}$ Department of Family Medicine, and ${ }^{7}$ Office of Technology Transfer, University of Michigan Medical School; and ${ }^{8}$ Department of Epidemiology, School of Public Health, University of Michigan, Ann Arbor, Michigan.
}

\section{Background}

The ascertainment of carriers of deleterious variants in cancer genes has increased since the isolation of BRCA1/2 in the mid-1990s. The U.S. Preventive Services Task Force (USPSTF) ${ }^{1}$ and NCCN, among others, recommend that a thorough family history be collected to identify hereditary syndromes. NCCN ${ }^{2,3}$ publishes evidence-based riskreduction guidelines and recommends that individuals who have an elevated cancer risk be referred for evaluation. Once identified, individuals have the potential to reduce their cancer risk by up to $95 \% .{ }^{4}$ However, most patients at risk for hereditary cancers remain unidentified. ${ }^{5,6}$ In Michigan, in $2015,{ }^{5}$ only $10.4 \%$ of unaffected women with a significant family history were referred for genetic counseling. In an integrated health system, ${ }^{6}<30 \%$ of patients had family history documented in the medical record. As a result, there is a pressing need to improve genetics clinics' efficiency, given the increasing number of patients being referred due to broadening testing guidelines, incidental identification of germline mutations through somatic tumor testing, and increasing awareness, despite a limited number of genetics providers.

To create an online tool to facilitate risk evaluation and guideline-based referrals, our team undertook a needs assessment in Michigan. The team interviewed healthcare providers about barriers to identifying highrisk patients and 2 issues surfaced: insufficient clinical time to complete a family history and data collected in the clinic lacking sufficient accuracy. Moreover, implementation of recommendations in NCCN Clinical Practice Guidelines in Oncology (NCCN Guidelines) was hampered by their complexity and frequent updates. The team sought to create a web-based, facile tool that would

See JNCCN.org for supplemental online content. 
enable patients to provide a complete personal and 3rd-degree family history in a setting where they could save the work and return. INHERET has the following characteristics to maximize its usefulness across diverse populations:

1. It is compatible with electronic devices, including smartphones;

2. It is written for English reading skills below 5th grade;

3. It covers all cancers; a drop-down menu for each family member enables the selection of diagnoses among 60 different cancer conditions and has features relevant to eligibility for risk evaluation (eg, Gleason grade in prostate cancer);

4. It collects 3rd-degree family history, including half-siblings, adopted individuals, and multiplets;

5. Data are saved while being input;

6. Data, owned by patients, are downloadable and portable;

7. Concise reports and recommendations follow best practice guidelines, graphic pedigree;

8. It links to educational content tailored to patients and providers; and

9. Reports can be integrated with medical record software.

Next, we set out to test it in silico, retrospectively and prospectively, in diverse settings: cancer genetics and primary care (PC) clinics. Figure 1 presents an overview of the creation and testing of INHERET.

\section{Patients and Methods}

The team obtained paper questionnaires used in genetics clinics, such as the Breast and Ovarian Cancer Risk Evaluation Clinic (BOCREC), to ascertain family history. The NCCN Guidelines ${ }^{2,3}$ and best practices were reviewed to ensure that INHERET collected all data elements required. A web-based form tied to a Microsoft SQL database housed on secure, HIPAA-compliant servers within the University of Michigan firewall was created.

Patients were invited by BOCREC schedulers via a mailed card or an email link to create an account and enter their data. In PC clinics, providers offered cards with a link. No patients were recruited by anyone with ties to INHERET, Inc.; informed consent was completed online.

\section{Results}

INHERET Testing

\section{In Silico and Retrospective Patient Histories}

The NCCN Guidelines for Genetic/Familial High-Risk Assessment ${ }^{2,3}$ in 2015 provided the primary foundation for a cognitive computing engine. For in silico testing, a program generated nearly every combination of cancer family structure to ensure INHERET's output was consistent with the NCCN Guidelines. Iterative testing continued until $100 \%$ concordance was achieved between INHERET and the NCCN Guidelines. More than 1 million test cases were evaluated before the tool was tested with real patient data.

Retrospective testing was completed using 347 historical patients from BOCREC and 78 manually generated patients to ensure broad representation of family structures. INHERET reports were $100 \%$ concordant when compared with genetic counselor clinical determinations.

\section{Preliminary Evaluation of INHERET Usability}

In 2017, 14 BOCREC patients consented (HUM00114820) to complete the INHERET questionnaire and provide feedback. We measured completion rate, having set a desired target of $70 \%$ a priori, based on experience with paper-based questionnaires, which had, before the inception of INHERET, been sent to patients before appointments were scheduled. We measured the accuracy of the reported results as determined by genetic counselor review and concordance with referral guidelines. Our target was $100 \%$ concordance between the history entered in INHERET and the verbal history conveyed by patients when queried by genetic counselors.

Ease of use and understandability were measured via follow-up surveys on a 5-point Likert scale (with $1=$ not at all easy to use/understand and $5=$ very easy to use/ understand) (see supplemental eAppendix 1, available with this article at JNCCN.org). Our target desirable performance was that $80 \%$ of patients would rank ease of use at $\geq 3$ (somewhat easy to use/easy to use/very easy to use) and $90 \%$ would rank understandability at $\geq 3$ (somewhat easy to understand/easy to understand/very easy to understand). Ease of use would be impacted by factors external to the INHERET tool, such as difficulties in collecting family health history from relatives, and by INHERET-specific factors. The understandability expectation was set at $90 \%$, having predicted that some patients may experience language barriers, especially because the Ann Arbor-area population includes immigrants from $>100$ countries. As shown in Figure 2, INHERET met or exceeded targets for completion rate and accuracy. However, it fell somewhat short on ease of use and understandability in this early test.

Based on this first series of usability tests, readability was reduced from a 9th-grade to a 4 th-grade level, navigation was simplified, smartphone compatibility across makers was added, and pedigree was improved, leading to INHERET version 2.0, the version that was pilot-tested prospectively in specialty and PC clinics. 

identifying which patients are at high risk

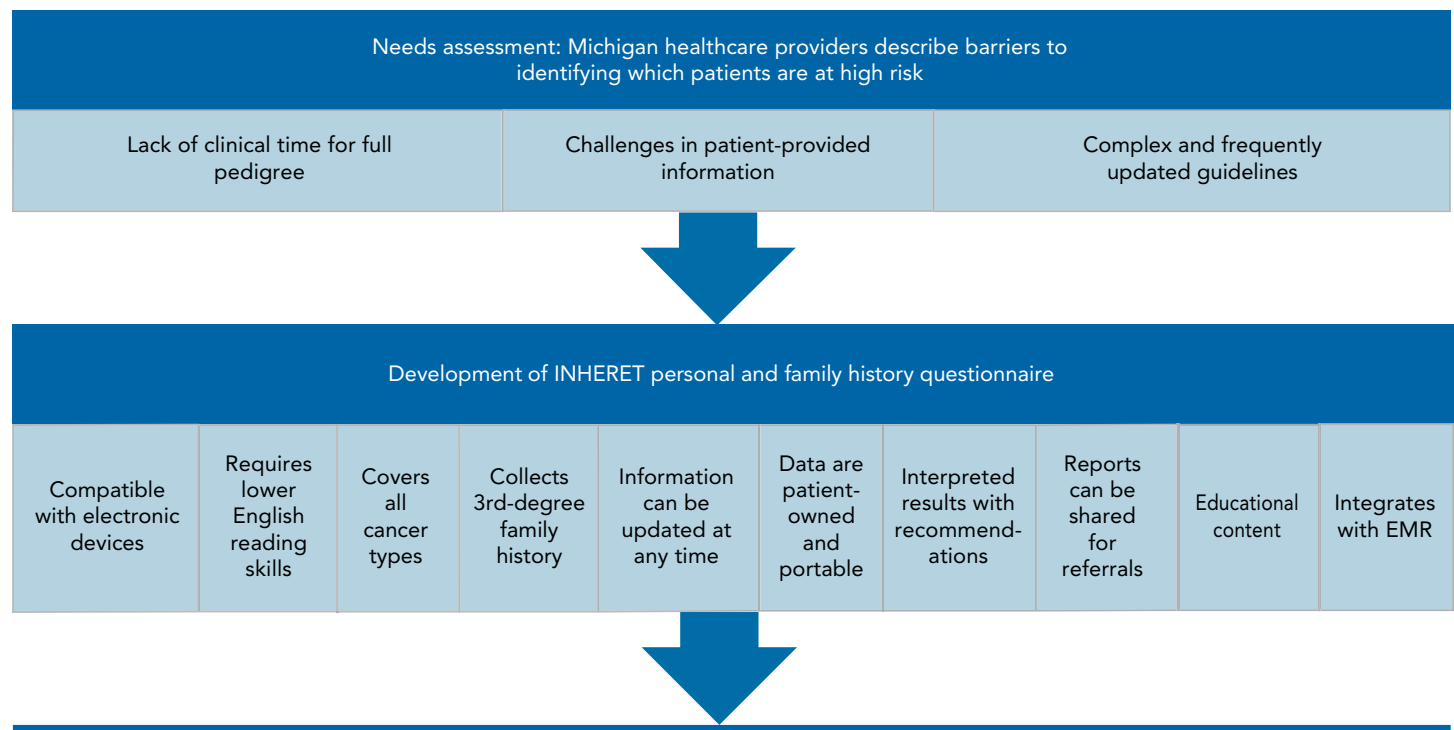

Prepilot test of INHERET: in silico, retrospective clinic cases, prospective cases

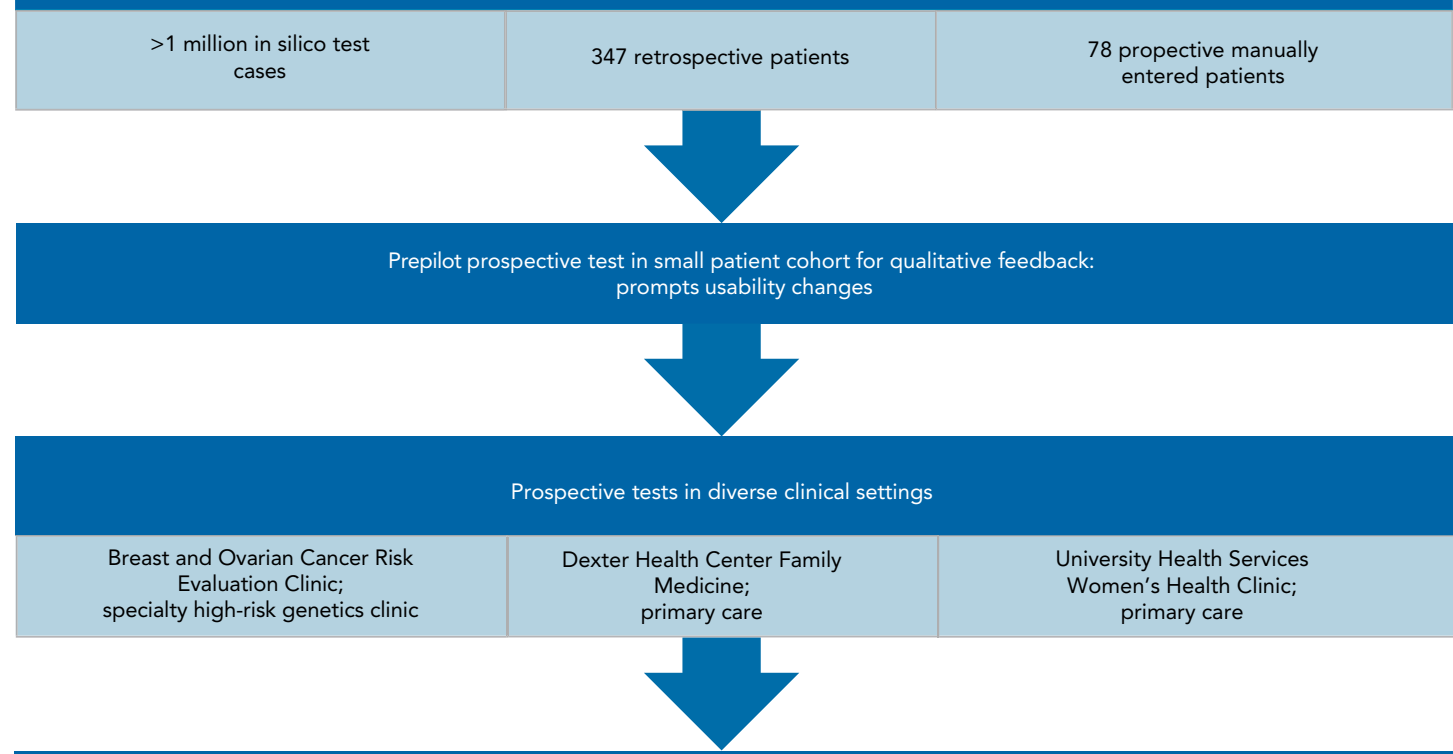

Qualitative focus group prompts further usability changes

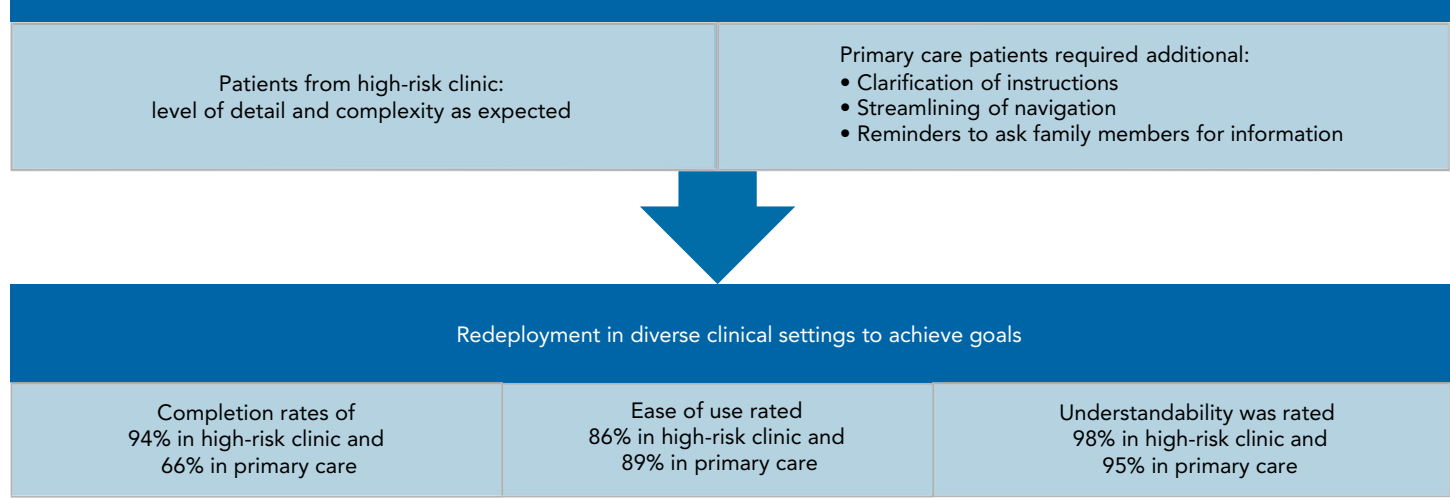

Figure 1. Trajectory of the creation, refinement, and testing of INHERET.

Abbreviation: EMR, electronic medical record. 


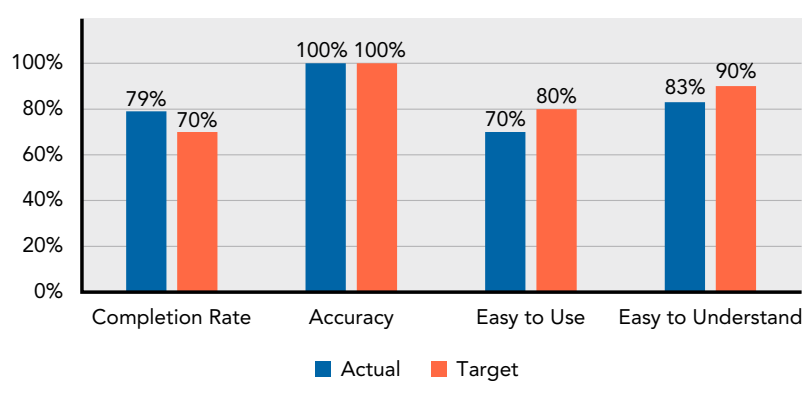

Figure 2. Completion and satisfaction in preliminary testing over time in a specialized genetics clinic (Breast and Ovarian Cancer Risk Evaluation Clinic). In 2017, initial feedback from patients $(n=14)$ showed achievement of targeted completion rates, accuracy, and ease of understandability but did not reach the $80 \%$ target in ease of use.

\section{Prospective Testing in Diverse Cancer Genetics and PC Clinics}

From October 2018 through December 2020, INHERET 2.0 was tested in 3 clinics under Institutional Review Board approval (HUM00138539): the BOCREC clinic (average age, 47 years), Dexter Health Center Family Medicine (DFM; PC; average age, 55 years), and University Health Services Women's Health Clinic (UHS; PC; female patients; average age, 31 years). Targets for success of the tool remained equivalent to the prior testing, except for an increase to $80 \%$ completion rates in BOCREC. We set a $50 \%$ completion rate target for PC because we surmised that PC patients would be less likely than BOCREC patients to be worried about cancer risk. In PC, the completion rates for surveys offered by the clinics were $70 \%$ (DFM) and $49 \%$ (UHS). Figure 3 depicts the evolution of completion results and satisfaction for BOCREC patients over time; all parameters show a steady improvement and surpassing of the target completion rate.

In light of these initial results, we undertook a second round of qualitative focus group interviews to refine INHERET. Through this process, we discovered that BOCREC patients, having been referred to a specialty clinic, were ready for the questionnaire's level of detail and complexity and were better prepared and motivated to enter their family and personal histories, given that they already knew they were likely to be at increased cancer risk. In contrast, PC patients required more direction and enhanced phone customer support. Further refinements based on patient suggestions were made to clarify navigation and add reminders that the patient could stop entering data and the work would be saved.

For the overall pilot, in Figure 3, we report the results from October 1, 2018, through December 31, 2020. During this period, 1,373 BOCREC patients were enrolled with an $85 \%$ completion rate, and 115 PC patients were enrolled with a 59\% completion rate (Figure 3A). Totals of 1,144 (83\%) BOCREC and 103 (54\%) PC patients completed both the family history questionnaire and the follow-up survey. Patient characteristics are reported in Table 1, including age, gender, frequency of INHERET results based on history and guideline algorithms, and racial/ethnic composition.

Overall, improvements to the INHERET questionnaire, navigation, pedigree, and adjustments for Applebased device compatibility resulted in steadily increasing completion and feedback rates. The quarterly data provided are cumulative rates and include all patient responses to the end of the quarter. Completion rates increased from $77 \%$ in 2019 to $82 \%$ in 2020 at BOCREC. In PC, they increased from $54 \%$ in 2019 to $67 \%$ in 2020 , with an overall completion rate of $59 \%$. Ease of use improved from $70 \%$ to $86.4 \%$ at BOCREC and from $45 \%$ to $89.5 \%$ at the PC sites, also exceeding target rates of $80 \%$ at each site. As anticipated, the ease of use score was impacted strongly by the effort required to research family health history, a variable highly dependent on family relationships and extrinsic to INHERET, with $19 \%$ of those surveyed commenting on this fact under the ease of use question. Understandability also showed significant increases, moving from $83 \%$ (Figure 2) to $97.8 \%$ (Figure 3C) at BOCREC and from $85 \%$ to $94.7 \%$ in PC (data not shown), exceeding the $90 \%$ target.

PC patients demonstrated a greater uptake at the UHS site $(n=96)$, which has a younger population (average age, 30 years) than the DFM site (average age, 56 years). However, the DFM patients were more likely to complete the questionnaire $(70 \%)$ than the UHS patients (57\%). Follow-up survey (concerning the usability of the tool) completion rates were $83 \%$ for BOCREC, $68 \%$ for DFM, and $51 \%$ for UHS, indicating that some of the patients providing data through INHERET chose not to answer the usability questions. A preliminary assessment of actions taken after the INHERET report revealed that, of 26 PC patients reported by INHERET to be eligible for referral for genetics, 19 (73\%) had so far been referred.

When ease of use and understandability data were examined based on educational achievement (Figure $4 \mathrm{~A})$, the differences were not significant. Age impacted ease of use scores, especially for patients aged $\geq 70$ years (average score, 2.6/5.0). No significant differences were noted in those between 20 and 69 years of age. Understandability was not impacted by age (Figure 4B).

The output of INHERET, in accordance with guidelines, consisted of a report that specified whether a patient met guidelines for referral for genetic counseling and further risk evaluation. In PC clinics, the rates of qualifying for further evaluation were $67 \%$ for DFP and $63 \%$ for UHS. For BOCREC, $78 \%$ of patients qualified for referral, which is, as expected, a high number, given that those patients had already been referred. Male patients 

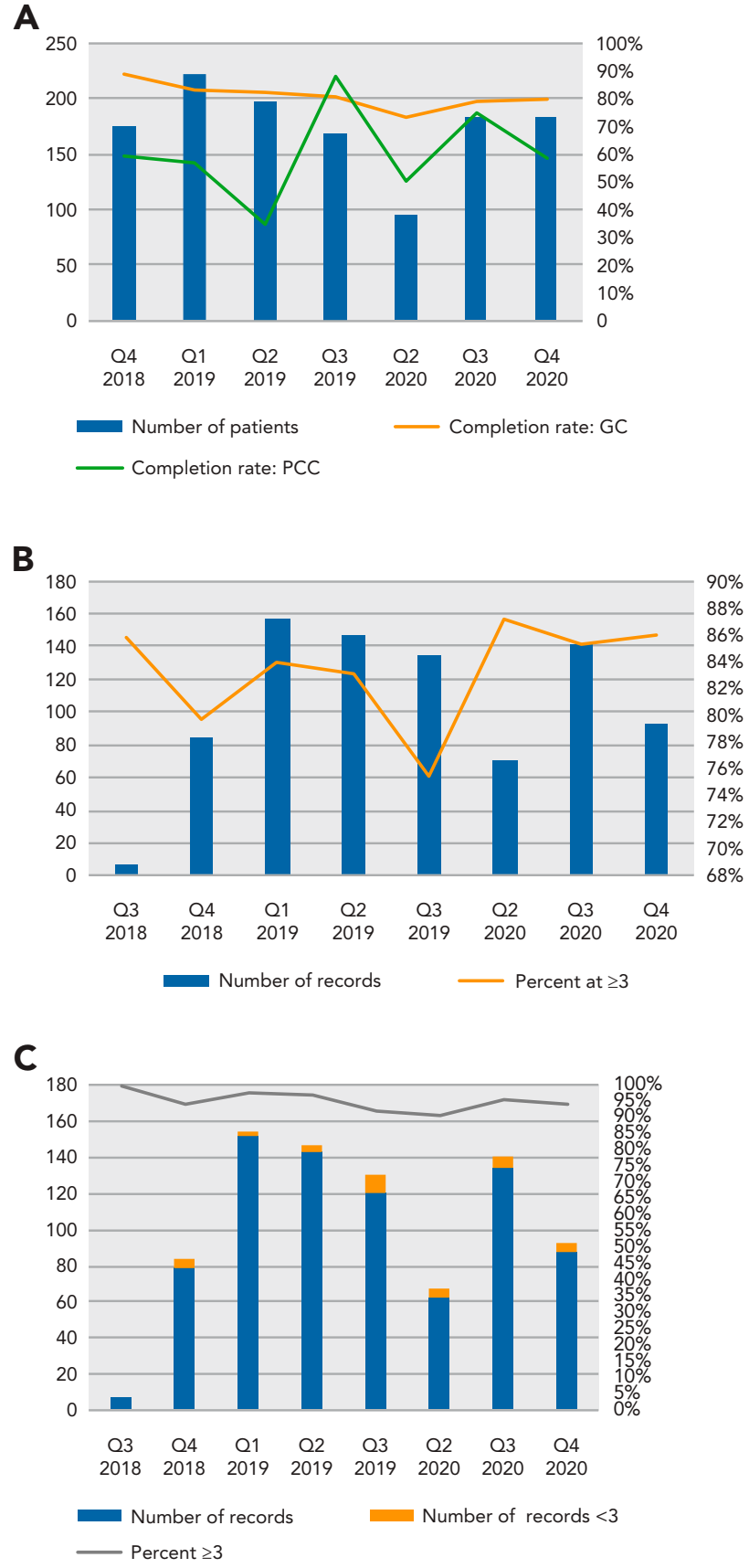

Figure 3. Overall results from pilot testing of INHERET over time. (A) Completion rates in genetics and primary care clinics. (B) Ease of use (scores of $\geq 3$ on a 5 -point scale). (C) Understandability (scores of $\geq 3$ on a 5 -point scale).

Note: 2019 Q4 and 2020 Q1 are omitted due to restructuring of clinics during the COVID-19 pandemic.

Abbreviations: GC, genetics clinic; $P C C$, primary care clinic; $Q$, quarter.

comprised $5.8 \%$ of the total number who completed the questionnaire, and their INHERET outputs were similar to those of women in PC, with $66 \%$ qualifying for referral, whereas $79 \%$ of women referred to BOCREC met guidelines for referral.
Physicians surveyed indicated that using INHERET in the clinic did not take extra time; $79 \%$ reported that they spent $<5$ minutes in collecting, reviewing, and discussing results with patients not appropriate for referral (Figure 5). Among patients appropriate for referral to genetics, $53 \%$ spent none to $<5$ minutes per patient in collecting, reviewing, and discussing results, and $33 \%$ spent between 5 and 9 minutes per patient.

Because this was an initial implementation project, during this pilot, INHERET was not yet integrated into the PC electronic medical record (EMR). The physician investigators unanimously indicated that they would prefer to receive their reports within the EMR system because it would streamline the process. Ninety-one percent responded that they were interested in continuing use of INHERET in the future, with that proportion increasing to $100 \%$ if INHERET were integrated with the EMR. Since the completion of the pilot, this integration has been completed and has been tested successfully in the clinic.

\section{Discussion}

\section{Effects of INHERET in a Specialized Genetics Clinic}

The BOCREC process for scheduling patients before INHERET was to mail a paper questionnaire, and $<70 \%$ of family histories were routinely completed. This process required multiple patient contacts over several weeks, delaying patient care. Thus, before the pilot testing of INHERET 2.0, the BOCREC queue was $>400$ patients. Up to $30 \%$ of referred patients dropped out altogether.

With the implementation of INHERET, patient throughput drastically improved: $82 \%$ of patients completed INHERET in 2020, compared with $<70 \%$ completion rate for mailed paperwork prior to implementation. The average turnaround time to completion was just 72 hours, reducing attrition to only $18 \%$, compared with $>30 \%$ pre-INHERET. By June 30,2019 , the queue was reduced from 400 to 15 patients, fewer than are seen during an average week. As an additional contribution to streamlining workflow, patients found by INHERET to be at population risk can be, for instance, counseled by genetic counselors by phone to emphasize population-based cancer screening or to review other concerns. Moreover, given the detailed information for each patient available through INHERET, prioritizing the patients who required appointments most urgently was facilitated.

Through INHERET, genetic counselors modified their templates to accommodate $20 \%$ more patients and completed their dictations during normal working hours. Taken together, the increased efficiencies of the cancer genetic counseling practice mediated by the implementation of INHERET resulted in genetic counselors increasing the number of evaluated patients by $50 \%$, if counting both 


\section{A}

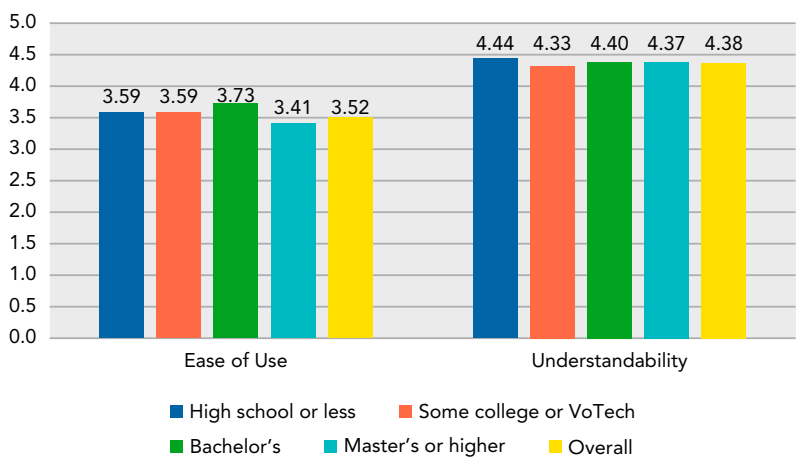

\section{B}

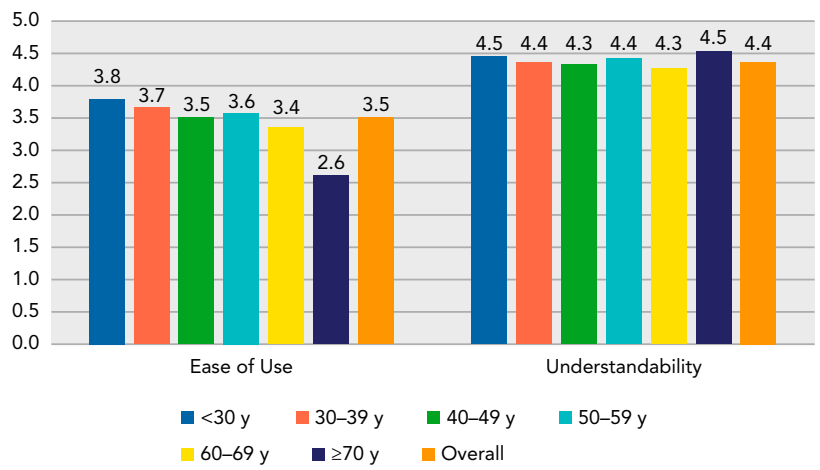

Figure 4. Ease of use and understandability versus (A) education level and (B) age at all sites.

Abbreviation: VoTech, vocational/technical education.

genetic counselor-only visits plus shared visits with the attending physician, without adding network hours to their week. During this implementation pilot, no other workflow changes occurred and there were no changes in the referral patterns or risk distribution of patients, so all the efficiencies gained can be ascribed to the use of INHERET.

Patients referred to BOCREC from all sites (PC, oncology or gynecology clinics, self-referrals, tumor board clinics, breast centers) were more likely to complete the INHERET questionnaire (85\%) than were PC patients (59\%). This is likely because appointments to BOCREC could not be scheduled without providing these data and because patients from PC sites, being, on average, at lower risk, may not have perceived filling out INHERET to be a priority. Given the COVID-19 pandemic-associated inducements to use technology in all forms of communication, learning, and healthcare, once BOCREC switched to telemedicine within 1 week of the country-wide shutdown in the United States, the completion rates for BOCREC remained high and were unaltered despite the deteriorating pandemic circumstances in the last 6 months of the pilot. This suggests the patients' increased understanding of the information needed to receive the most comprehensive care by telemedicine during unusual times. INHERET provided an accessible way to fill the information gap and thus facilitated telemedicine counseling.

INHERET's use in BOCREC streamlined the workflow and improved efficiencies. Schedulers reduced the number of calls needed to remind patients to obtain their family histories and return forms. Schedulers emailed links to patients through the INHERET portal, singly or in batches, and the reports were returned in an average of 72 hours; schedulers expressed enthusiasm for the tool compared with the prior workflow. Patients aged $<70$ years found the online process to be preferable to the paper form, as evidenced by the significantly higher completion rate $(94 \%$ vs $70 \%)$ than for the eldest age group, which comprised $4 \%$ of total patients.

\section{Effects of INHERET in PC Clinics}

In PC clinics, $>59 \%$ of patients who began the family history questionnaire completed it, with $100 \%$ completing it in the last 6 months. Ease of use increased from $45 \%$ to $87 \%$ of patients finding INHERET easy to use, whereas $92 \%$ found it easy to understand. Of the 131 total PC patients, 86 completed the questionnaire, and $49 \%$ were identified for referral to genetics. This is likely due to self-guided selfselection, because only between $10 \%$ to $20 \%$ of patients are estimated to be eligible for referral by guidelines. Before INHERET, PC clinics had no systematic way to ascertain high-risk patients. Based on this experience, we surmise that, when implemented in PC settings, patients will selfselect by perceived or known prior risk, as we speculate may have occurred during this study. We believe our data confirm that those patients who think they may have a family history indicative of increased risk will more readily complete INHERET, whereas those who are less aware or do not believe their history is relevant may not.

INHERET proved especially useful for established patients who were not previously identified to be at increased risk in PC. The high-risk patients ascertained through INHERET would now be made aware of their risk and would pursue appropriate downstream care. INHERET provides a thorough list of the reasons that confer increased risk and thus facilitates evidence-based justification for counseling on tailored enhanced strategies.

The high INHERET completion rates were seen by the PC providers as a sign that a major proportion of patients not previously identified as being at increased risk can now be referred for specialized genetic evaluation. This was accomplished without impinging on the PC clinic's workflow or increasing physician time spent on the encounter, both primary objectives of INHERET since its conception.

The integration of INHERET to enable high-quality clinical services in cancer risk management 


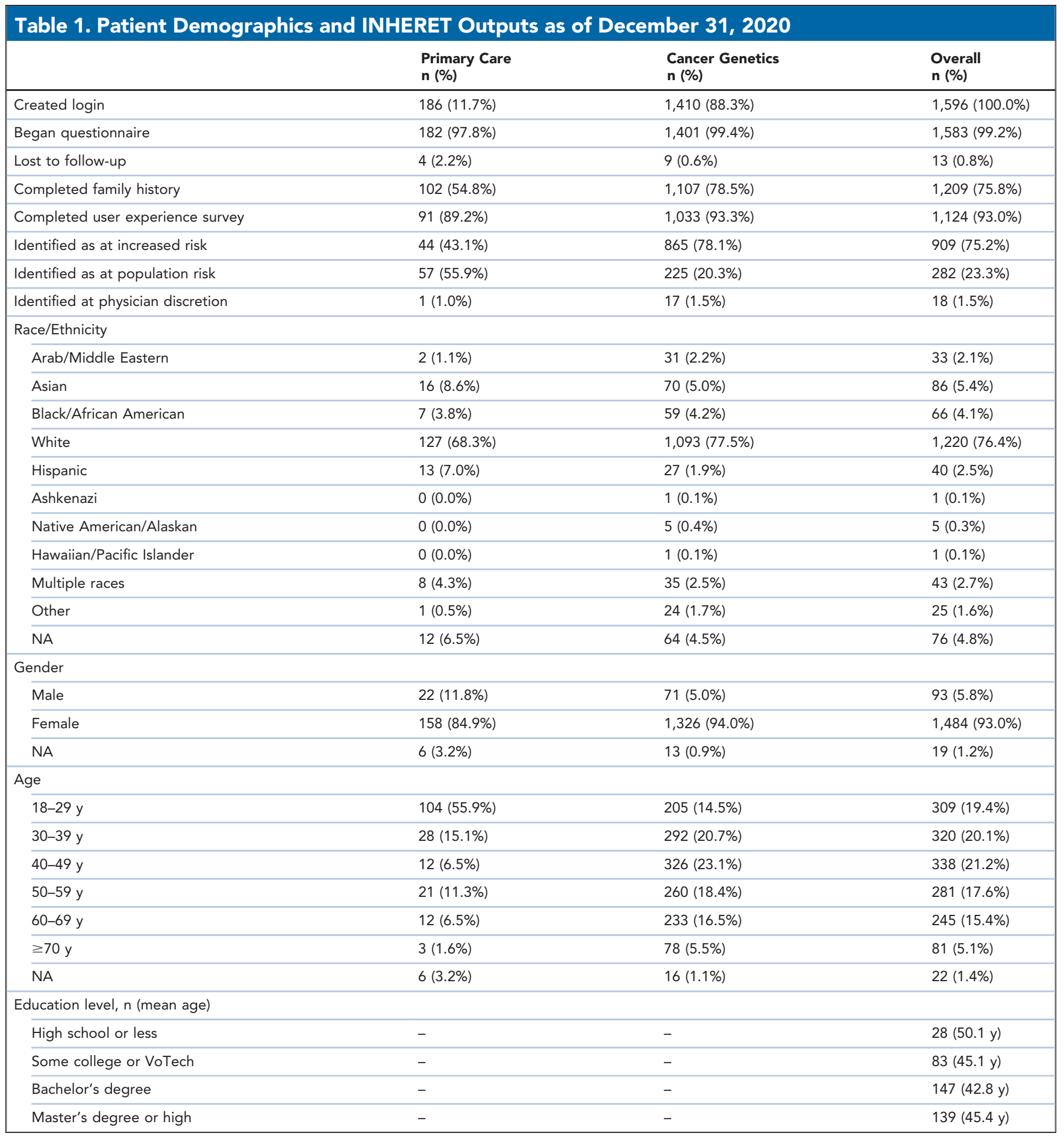

Abbreviations: NA, not available; VoTech, vocational/technical education.

presented challenges. Early in the pilot, we discovered that some patients did not know the name of the healthcare clinic or of their physician, even when the names were in a dropdown list. Thus, we implemented an invitation process whereby the provider's office sends a secure email invitation to the patient with an embedded code tied to the patient's demographic data and the clinic and physician who receive the report, without revealing patient health information. INHERET may not be the optimal method to collect data from a small fraction of individuals aged $>70$ years, who may require alternative methods. 


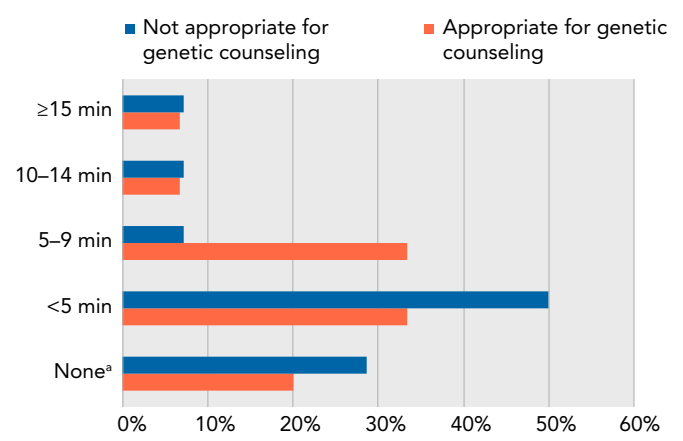

Figure 5. Use of clinician time spent with patients in primary care clinics to convey results of INHERET.

alt was handled by my staff.

\section{Conclusions}

Use of INHERET enhanced throughput in genetics clinics, increasing efficiency of counselors and thus freeing more time to directly care for more patients, essentially eliminating loss to follow-up with the inception of widespread telemedicine in cancer genetics due to the COVID-19 pandemic.

In PC, using INHERET did not cause a time burden on providers. INHERET facilitates family history interpreted against practice guidelines with clear recommendations and simplifies the downstream care for patients at increased risk. Providers unanimously found INHERET to be valuable for patient care.
Submitted March 6, 2021; final revision received June 10, 2021; accepted for publication June 10, 2021.

Author contributions: Study design: Schroeder. Questionnaire and/or report design: Milliron, Merajver. Data assembly: McCain. Data analytic support: Schroeder. Program design and/or development: McCain, Arthurs, Schroeder, Keren, Merajver. Reviewed program testing results: McCain. Patient enrollment: Kittendorf, Harper. Patient data entry: Cook. Reviewed outputs: Milliron, Keren, Merajver. Programmer of INHERET tool: Paquette. Tool validation: Merajver. Reviewed guidelines and interpretation for coding: Milliron, Hulswit, Merajver. Guideline coding system creation: Cook. Coded guidelines into INHERET: Cook. Scientific lead: Merajver. Lead, pilot testing of INHERET: Parvaz, Ernst, Zazove. Clinical pilot in Breast and Ovarian Cancer Risk Evaluation Clinic: Milliron, Hulswit. Managed pilot testing for site: Merajver. Managed INHERET development team: Keren. Help desk for patients and providers: Tippie. Problem reporting and resolution: Tippie. Provided anonymized data for reporting: Tippie. Writing: McCain, Milliron, Cook, Kittendorf, Harper, Tippie, Arthurs, Hulswit, Keren, Merajver.

Disclosures: Ms. McCain, Ms. Milliron, Ms. Cook, Mr. Paquette, Mr. Arthurs, Mr. Tippie, Ms. Hulswit, Dr. Schroeder, Dr. Keren, and Dr. Merajver are affiliated with INHERET, Inc. and receive payment for their services and/ or are equity holders in the company. The remaining authors have disclosed that they have no financial interests, arrangements, affiliations, or commercial interests with the manufacturers of any products discussed in this article or their competitors.

Funding: Research reported in this publication was partially funded by a grant from the Blue Cross Blue Shield of Michigan Foundation (N024454 UMF; D.F. Keren); a phase I STTR grant from the NIH under award number 1R42CA239842-01 (D.F. Keren); and funds from the Breast Cancer Research Foundation, the Rogel Cancer Center (NIH grant P30CA046592), the Ravitz Foundation, the Michigan Emerging Technologies Fund, and Ann Arbor SPARK.

Disclaimer: The content is solely the responsibility of the authors and does not necessarily represent the official views of the $\mathrm{NIH}$.

Correspondence: Sofia D. Merajver, MD, PhD, University of Michigan Rogel Cancer Center, 1500 East Medical Center Drive, Room 7314, Ann Arbor, Ml 48109-5948. Email: smerajve@umich.edu

\section{References}

1. U.S. Preventive Services Task Force. Final recommendation statement: BRCA-related cancer risk assessment, genetic counseling, and genetic testing. Accessed June 12, 2019. Available at: https://www. uspreventiveservicestaskforce.org/uspstf/recommendation/brca-relatedcancer-risk-assessment-genetic-counseling-and-genetic-testing

2. Daly MB, Pilarski R, Berry MP, et al. NCCN Clinical Practice Guidelines in Oncology for Detection, Prevention, and Risk Reduction: Genetic/ Familial High-Risk Assessment: Breast and Ovarian. Version 3.2019. Accessed June 12, 2019. To view the most recent version, visit NCCN.org

3. Provenzale D, Gupta S, Ahnen D, et al. NCCN Clinical Practice Guidelines in Oncology for Detection, Prevention, and Risk Reduction: Genetic/Familial High-Risk Assessment: Colorectal. Version 1.2019. Accessed June 12, 2019. To view the most recent version, visit NCCN.org
4. Hartmann LC, Lindor NM. The role of risk-reducing surgery in hereditary breast and ovarian cancer. N Engl J Med 2016;374: 454-468.

5. Fussman C, Schrager J, Duquette D. Breast and ovarian cancer personal/ family history and genetic counseling utilization among Michigan women. Michigan BRFSS Surveillance Brief. Vol. 10, No. 3. Lansing, MI: Michigan Department of Health and Human Services, Lifecourse Epidemiology and Genomics Division, December 2016.

6. Knerr S, Bowles EJA, Leppig KA, et al. Trends in BRCA test utilization in an integrated health system, 2005-2015. J Natl Cancer Inst 2019;111:795-802.

7. Ramsey SD, Yoon P, Monsinge R, Khoury MJ. Population-based study of the prevalence of family history of cancer: implications for cancer screening and prevention. Genet Med 2006;8:571-575. 
Supplemental online content for:

\section{Implementation of INHERET, an Online Family History and Cancer Risk Interpretation Program for Primary Care and Specialty Clinics}

Lynn A. McCain, MHSA; Kara J. Milliron, MS, CGC; Amanda M. Cook, BA; Robert Paquette, BS; Jasmine B. Parvaz, MD, PhD; Susan D. Ernst, MD; Anne L. Kittendorf, MD; Diane M. Harper, MD; Philip Zazove, MD; Jim Arthurs, BSEE; Jerry A. Tippie, BA; Bailey Hulswit, MS, CGC;

Lee F. Schroeder, MD, PhD; David F. Keren, MD; and Sofia D. Merajver, MD, PhD

J Natl Compr Canc Netw 2022;20(1):63-70

eAppendix 1: INHERET Questionnaire Survey Scale 


\section{eAppendix 1. INHERET Questionnaire Survey Scale}

1. How easy was it to use the Family Health Questionnaire?

Not at all

easy to use

1
Somewhat

easy to use

3

4

Comments

2. Did you understand the questions?

$\begin{array}{cc}\begin{array}{c}\text { Not at all } \\ \text { easy to }\end{array} & \begin{array}{c}\text { Somewhat } \\ \text { easy to } \\ \text { understand }\end{array} \\ \text { understand }\end{array}$

understand

1 understand

3
Very

easy to use

5

Comments

3. Do you have any additional comments about the Family Health Questionnaire that you would like to share with us? 